\title{
Artificial Boundary Conditions for the Linear Advection Diffusion Equation
}

\author{
By Laurence Halpern
}

\begin{abstract}
A family of artificial boundary conditions for the linear advection diffusion equation with small viscosity is developed. Well-posedness for the associated initial boundary value problem is analyzed. The error produced by truncating the domain is estimated. Numerical results are presented.
\end{abstract}

1. Introduction. When computing the solution of a partial differential equation in an unbounded domain, one often introduces artificial boundaries. In order to limit the computational cost, these boundaries must be chosen not too far from the domain of interest. Therefore, the boundary conditions must be good approximations to the so-called "transparent" boundary condition (i.e., such that the solution of the problem in the bounded domain is equal to the solution in the original domain). The transparent boundary condition is usually an integral relation in time and space between $u$ and its normal derivative on the boundary, which makes it impractical from a numerical point of view. One must approximate this relation to get local boundary conditions: they are often called absorbing or artificial boundary conditions.

This question is of crucial interest in such different areas as geophysics, plasma physics, fluid dynamics [1], [2], [3], and the use of such conditions is now classical in geophysics.

Our interest for the linear advection diffusion equation comes from the NavierStokes equation, but it arises also in other fields as, for example, meteorology [6].

The incompressible Navier-Stokes equation can be written as

$$
\begin{aligned}
& \mathbf{u}_{t}+(\mathbf{u} \cdot \nabla) \mathbf{u}-\nu \Delta \mathbf{u}+\nabla p=0, \\
& \operatorname{div} \mathbf{u}=0,
\end{aligned}
$$

where $\nabla$ is the gradient operator and $\Delta$ the Laplacian. The viscosity $\nu$ is assumed to be small.

A common application is the flow around a body. Far away, the flow $\mathbf{u}$ is almost constant, equal to a [7]. Linearizing the equation, and using a vorticity formulation yields

$$
\omega_{t}+(\mathbf{a} \cdot \nabla) \omega-\nu \Delta \omega=0
$$

which is the equation we are dealing with in this work.

Received February 14, 1985.

1980 Mathematics Subject Classification. Primary 35A35, 35K22, 41A21.

(C)1986 American Mathematical Society $0025-5718 / 86 \$ 1.00+\$ .25$ per page 
We assume that $a_{1}$ is positive, so that the solution is essentially propagating in the right $x$-direction.

We further assume that the data are of compact support in space. We put artificial boundary conditions at $x_{2}$ and $x_{1}$, and find "good" boundary conditions.

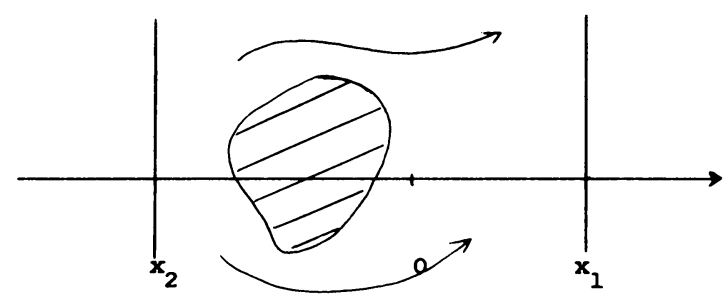

Because of the rightward propagation, the conditions to impose on the right and left boundaries, respectively, are inherently different. In the first five sections we deal with the right boundary.

In Section 2, using as essential tool the Fourier transform, we formulate the transparent boundary condition and approximate it for small values of the viscosity by means of generalized continued fractions. This leads to an infinite family of boundary conditions, which are partial differential equations of first order in $x$. For example, in one dimension, the first three boundary conditions are

$$
\begin{aligned}
& \omega_{x}=0 \text {, } \\
& \omega_{t}+a \omega_{x}=0, \quad(a \neq 0) . \\
& \omega_{t}+a \omega_{x}+\nu\left(2 a \omega_{x t}+\omega_{t t}\right)=0,
\end{aligned}
$$

Relations (1.3) and (1.4) are a Neumann and transport condition, respectively. They are easy to guess and have already been used in applications. As far as we know, the higher-order conditions are new.

In Section 3 we analyze these boundary conditions and give energy estimates, which show that the associated initial boundary value problems are well-posed.

In Section 4, using again the Fourier transform, we establish $L^{2}$-norm error estimates for the solution in the slab $\left[0, x_{1}\right]$, in terms of $x_{1}, \nu$, and the original solution in $[0,+\infty[$ : the accuracy of the $n$th order boundary condition is of order $\nu^{2 n}$.

In Section 5 we give numerical schemes which discretize the equation and the right-hand boundary conditions, and we show by numerical results the efficiency of the approximation.

In Section 6 we briefly develop the boundary conditions for the left boundary $x_{2}$. The results analogous to those in Sections 3 and 4 are given without proofs, since the proofs are essentially the same as for the boundary on the right.

2. Construction of a Family of Absorbing Boundary Conditions. To begin with, we formulate at every point $x$ outside the support of the data an integro-differential equation, which will in time be the transparent boundary condition.

2.1. The transparent boundary condition. We consider the solutions of the equation

$$
L_{\nu} u=0
$$


in the halfspace $x \geqslant 0$, where $L_{\nu}$ is the differential operator

$$
L_{\nu}=\frac{\partial}{\partial t}+\frac{\partial}{\partial x}+\mathbf{a} \cdot \nabla-\nu \Delta .
$$

The vector a is given in $R^{n}, \nabla$ denotes the gradient with respect to $\mathbf{y}=\left(y_{1}, \ldots, y_{n}\right)$ and $\Delta$ the Laplacian in all variables $\mathbf{X}=(x, \mathbf{y})$.

THEOREM 1. The transparent boundary condition at point $x_{1}$ is

$$
\frac{\partial u}{\partial x}=\iint \lambda(\mathbf{k}, \omega) \hat{u}\left(x_{1}, \mathbf{k}, \omega\right) e^{i(\omega t+\mathbf{k} \cdot \mathbf{y})} d \omega d \mathbf{k},
$$

where $\lambda$ is given by

$$
\begin{aligned}
& \lambda=\left(1-\delta^{1 / 2}\right) / 2 \nu, \\
& \delta=1+4 i \nu(\omega+\mathbf{a} \cdot \mathbf{k})+4 \nu^{2}|\mathbf{k}|^{2} .
\end{aligned}
$$

The determination of $\delta^{1 / 2}$ is chosen such that

$$
\operatorname{Re} \delta^{1 / 2}>0 \text {. }
$$

Proof. We shall write at every point $x \geqslant 0$ an integral relation between $u$ and $u_{x}$. For this purpose, we use the Fourier transform in $t$ and $\mathbf{y}$. The Fourier transform $\hat{u}$ of every solution $u$ to $L_{\nu}$ in the halfspace $x \geqslant 0$ satisfies the second-order ordinary differential equation in $x$ :

$$
-\nu \hat{u}_{x x}+\hat{u}_{x}+\left(i(\omega+\mathbf{a} \cdot \mathbf{k})+\nu|\mathbf{k}|^{2}\right) \hat{u}=0 .
$$

The solutions of this equation have the form

$$
\hat{u}(\omega, x, \mathbf{k})=\hat{a}(\omega, \mathbf{k}) e^{\lambda x}+\hat{b}(\omega, \mathbf{k}) e^{\lambda^{\prime} x},
$$

where $\lambda$ and $\lambda^{\prime}$ solve the quadratic equation

$$
-\nu \lambda^{2}+\lambda+i(\omega+\mathbf{a} \cdot \mathbf{k})+\nu|\mathbf{k}|^{2}=0 .
$$

The discriminant is $\delta$, given by (2.5). One root is $\lambda$ (cf. (2.4)), the other is

$$
\lambda^{\prime}=\left(1+\delta^{1 / 2}\right) / 2 \nu
$$

Our choice (2.6) implies that the real part of $\lambda$ is negative, while that of $\lambda^{\prime}$ is positive. In order to keep $\hat{u}$ bounded in the halfplane $x \geqslant 0$, the coefficient $\hat{b}$ must vanish, so that

$$
\hat{u}(x, \mathbf{k}, \omega)=\hat{a}(\mathbf{k}, \omega) e^{\lambda(\mathbf{k}, \omega) x} .
$$

By differentiation with respect to $x$, the following identity is seen to hold at every point $x \geqslant 0$ :

$$
\frac{\partial \hat{u}}{\partial x}-\lambda \hat{u}=0 .
$$

Hence, at point $x_{1}, u$ satisfies the integro-differential equation (2.3).

Relation (2.3) is the transparent boundary condition at point $x_{1}$. It is global in time and space. Hence we shall approximate the nonlocal operator

$$
u \rightarrow \iint \lambda(\mathbf{k}, \omega) \hat{u}(x, \mathbf{k}, \omega) e^{i(\omega t+\mathbf{k} \cdot \mathbf{y})} d \omega d \mathbf{k}
$$

by a suitably chosen local operator, in time and space. 
2.2. Approximation of the transparent boundary condition. For wave equations, the approximation is made with respect to the incidence angle to the boundary, that is, roughly, in terms of $|\mathbf{k}| / \omega$. The strategy here is different: we shall approximate $\lambda(\mathbf{k}, \omega)$ for small values of $\nu$, for any value of $\mathbf{k}$ and $\omega$. In order to get a local operator, we shall use polynomial or rational approximations.

Taylor approximation. The first two approximations to $\lambda$, given by (2.4), come from zero- and first-order Taylor approximation to $\delta^{1 / 2}$, respectively:

$$
\left\{\begin{array}{l}
\delta^{1 / 2}=1+O(\nu) \\
\delta^{1 / 2}=1+2 i \nu(\omega+\mathbf{a} \cdot \mathbf{k})+O\left(\nu^{2}\right)
\end{array}\right.
$$

which give

$$
\left\{\begin{array}{l}
\lambda=O(1) \\
\lambda=-(\omega+\mathbf{a} \cdot \mathbf{k})+O(\nu)
\end{array}\right.
$$

These approximations lead to the boundary conditions

$$
\begin{aligned}
& B_{0} u=u_{x}=0, \\
& B_{1} u=u_{t}+u_{x}+\mathbf{a} \cdot \nabla u=0 .
\end{aligned}
$$

Higher-order approximations. For higher-order approximations, various strategies can be applied. The idea of using Taylor or Padé approximations has been introduced for the wave equation in [1]. There, the authors have shown that, except for the first one, Taylor approximations lead to ill-posed problems, while a hierarchy of Padé approximants lead to well-posed problems. We shall develop here a family of generalized continued fractions related to certain Padé approximants.

It is well-known (see [4]) that one can approximate a root $\lambda$ of a quadratic equation

$$
a \lambda^{2}+b \lambda+c=0
$$

in terms of generalized continued fractions by rewriting the equation as

$$
\lambda(a \lambda+b+d)+c-\lambda d=0
$$

and defining the sequence $\lambda_{n}$ by

$$
\lambda_{n+1}=\left(\lambda_{n} d-c\right) /\left(a \lambda_{n}+b+d\right)
$$

for a suitable choice of $d$. We choose here $d$ and $\lambda_{1}$ as follows:

$$
\left\{\begin{array}{l}
\lambda_{1}=-i(\omega+\mathbf{a} \cdot \mathbf{k}) \\
d=-i \nu(\omega+\mathbf{a} \cdot \mathbf{k})
\end{array}\right.
$$

Thus, as a function of $\nu, \lambda_{n}$ is the quotient of two polynomials of degree $n-1$, $P_{n-1}$ and $Q_{n-1}$, given recursively by

$$
\left\{\begin{array}{l}
P_{n}=-i(\omega+\mathbf{a} \cdot \mathbf{k}) \nu P_{n-1}+\left(i(\omega+\mathbf{a} \cdot \mathbf{k})+\nu|\mathbf{k}|^{2}\right) Q_{n-1}, \\
Q_{n}=\nu P_{n-1}-(1+i(\omega+\mathbf{a} \cdot \mathbf{k}) \nu) Q_{n-1} .
\end{array}\right.
$$

Furthermore, $\lambda_{n}$ is of order $2 n-1$ in $\nu$,

$$
\lambda_{n}-\lambda=O\left(\nu^{2 n-1}\right) \text {. }
$$


In other words, $\lambda_{n}$ is the $[(n-1) /(n-1)]$ Padé approximant to $\lambda$. This can be proved by defining the error coefficient $q_{n}$ as

$$
q_{n}=\left(\lambda-\lambda_{n}\right) /\left(\lambda^{\prime}-\lambda_{n}\right) .
$$

An easy calculation shows that

$$
\left\{\begin{array}{l}
q_{n}=\left(q_{1}\right)^{n} \\
q_{1}=O\left(\nu^{2}\right)
\end{array}\right.
$$

and the estimate for $\lambda_{n}$ follows.

Remark 1. What first comes to mind, when seeking a sequence of rational fractions approximating $\lambda$, would be to choose $d=0$ (and $\lambda_{1}=0$ ). Unfortunately, this provides a less accurate approximation $(n-1, n)$, with $\lambda_{n}-\lambda=O\left(\nu^{n-1}\right)$. Likewise, the choice $d=1$ leads to a divergent sequence $\lambda_{n}$.

Boundary operators. We return to $\lambda_{n}$ as a function of $\nu, \omega$ and $\mathbf{k}$. It is easy to see inductively that $P_{n}$ and $Q_{n}$ are also polynomials in $\omega$ and $\mathbf{k}$, and their degrees in these variables are $n+1$ and $n$, respectively. The corresponding approximation to the boundary identity (2.3) is in Fourier coordinates

$$
B_{n} \hat{u}=0 \text {, }
$$

where $B_{n}$ is given by

$$
B_{n}=Q_{n-1}(\mathbf{k}, \omega) \frac{\partial}{\partial x}-P_{n-1}(\mathbf{k}, \omega) .
$$

Upon application of the inverse Fourier transform, this formula yields a local operator $B_{n}$ which is globally of order $n$, and of order 1 in $x$.

Example. The second-order boundary operator is

$$
B_{2}=-B_{1}-\nu B_{y}\left(\frac{\partial}{\partial x}+B_{1}\right)+\nu \Delta_{y}
$$

where $\Delta_{y}$ is the Laplacian with respect to $y$, and $B_{y}$ the transverse transport operator

$$
B_{y}=\frac{\partial}{\partial t}+\mathbf{a} \cdot \nabla
$$

For the study of the associated initial boundary value problems, a formal factorization of the boundary operators $B_{n}$ will be useful.

LEMMA 1. If $u$ is a sufficiently smooth solution to the problem

$$
\begin{aligned}
& L_{\nu} u=0 \quad \text { for } x \leqslant x_{1}, \\
& B_{n} u\left(x_{1}, \mathbf{y}, t\right)=0,
\end{aligned}
$$

then $u$ satisfies the boundary condition

$$
\left(B_{1}\right)^{n} u\left(x_{1}, \mathbf{y}, t\right)=0 .
$$

Proof. The hypothesis can be written in Fourier coordinates as in (2.7),

$$
\begin{aligned}
& -\nu \hat{u}_{x x}+\hat{u}_{x}+\left(i(\omega+\mathbf{a} \cdot \mathbf{k})+\nu|\mathbf{k}|^{2}\right) \hat{u}=0, \\
& B_{n} \hat{u}=Q_{n} \hat{u}_{x}-P_{n} \hat{u}=0, \quad x=x_{1} .
\end{aligned}
$$


Using the recurrence formulae for $P_{n}$ and $Q_{n}$, we get

$$
B_{n}=-\nu\left(\frac{\partial}{\partial x}+i(\omega+\mathbf{a} \cdot \mathbf{k})\right) B_{n-1}
$$

then we have on the boundary

$$
B_{n}=(-\nu)^{n-1}\left(B_{1}\right)^{n} \text {. }
$$

3. A Priori Estimates for the Initial Boundary Value Problem. We consider the following problem in the halfspace $x \leqslant x_{1}$ :

$$
\begin{cases}L_{\nu} u=0, & x \leqslant x_{1}, t \in[0, T], \\ u(\mathbf{X}, 0)=u^{0}, & x \leqslant x_{1}, \\ B_{n} u\left(x_{1}, \mathbf{y}, t\right)=0, & t \in[0, T] .\end{cases}
$$

We assume $u^{0}$ to be as smooth as needed, with compact support in the halfspace $\Omega_{-}=\left\{X ; x \leqslant x_{1}\right\}$. Then the problem is well-posed, i.e., there are a priori estimates:

THEOREM 2. Suppose $u$ is a solution to (3.1). Then the following holds:

(i) $u$ belongs to $L^{\infty}\left(0, T, L^{2}\left(\Omega_{-}\right)\right) \cap L^{2}\left(0, T, H^{1}\left(\Omega_{-}\right)\right)$;

(ii) $u$ belongs to $L^{\infty}\left(0, T, L^{2}\left(R^{n-1}\right)\right)$ on the boundary $x=x_{1}$.

Before proving the theorem, we introduce some notations.

We denote by $q, q_{1}, q_{0}$ the squares of the usual Sobolev norms (or seminorms), defined by

$$
\begin{aligned}
q(u) & =\iint u^{2}(x, \mathbf{y}, t) d x d \mathbf{y} \\
q_{1}(u) & =\iint|\nabla u|^{2}(x, \mathbf{y}, t) d x d \mathbf{y} \\
q_{0}(u) & =\int u^{2}\left(x_{1}, \mathbf{y}, t\right) d \mathbf{y} .
\end{aligned}
$$

Proof of Theorem 2. Suppose $u$ is a solution to $L_{\nu} u=0$ in the halfspace, that is,

$$
u_{t}+u_{x}+\mathbf{a} \cdot \nabla u-\nu \Delta u=0 \text {. }
$$

Multiplying by $u$ and integrating with respect to $\mathbf{X}$ in the whole domain, we get

$$
\frac{d}{d t} q(u)+2 \nu q_{1}(u)+q_{0}(u)-2 \nu \int\left(u u_{x}\right)\left(x_{1}, \mathbf{y}, t\right) d \mathbf{y}=0 .
$$

The condition $B_{0}$ is quite easy to analyze: If $u_{x}$ vanishes on the boundary, the last term in (3.3) is zero, and the result is clear. If $n$ is equal to 1 , then $u$ satisfies the boundary condition

$$
u_{t}+u_{x}+\mathbf{a} \cdot \nabla u=0
$$

we can replace $u_{x}$ in the last term of (3.3),

$$
\frac{d}{d t}\left(q(u)+\nu q_{0}(u)\right)+2 \nu q_{1}(u)+q_{0}(u)=0,
$$

which gives estimates (i) and (ii) in this case.

We now proceed by induction: Assume the theorem holds for $B_{n-1}$. If the function $u$ is a solution of (3.1), we define a function $v$ for $x \leqslant x_{1}, t$ in $[0, T]$, by

$$
v=u_{t}+u_{x} .
$$


The function $v$ is a solution of (3.1) for $n-1$ and therefore fulfills (i) and (ii). Furthermore, multiplying (3.5) by $u$ and integrating in $\mathbf{y}$ for $x$ equal to $x_{1}$, we get

$$
\frac{d}{d t} q_{0}(u)+2 \int\left(u u_{x}\right)\left(x_{1}, \mathbf{y}, t\right) d \mathbf{y}=2 \int(u v)\left(x_{1}, \mathbf{y}, t\right) d \mathbf{y}
$$

We multiply (3.6) by $\nu$ and add it to (3.3) to get rid of the last term in the left-hand side:

$$
\frac{d}{d t}\left(q(u)+\nu q_{0}(u)\right)+2 \nu q_{1}(u)+q_{0}(u)=\nu \int(u v)\left(x_{1}, \mathbf{y}, t\right) d \mathbf{y}
$$

We now use the following inequality on the right-hand side,

$$
u v \leqslant \varepsilon u^{2}+v^{2} / 4 \varepsilon .
$$

By choosing $\varepsilon$ such that $\nu \varepsilon \leqslant \alpha<1$, we finally obtain

$$
\frac{d}{d t}\left(q(u)+\nu q_{0}(u)\right)+2 \nu q_{1}(u)+C q_{0}(u) \leqslant q_{0}(v),
$$

which gives the desired bounds.

4. Error Estimates for the Approximate Problem. Let us consider the initial boundary value problem in the halfspace $x \geqslant 0$ :

$$
\begin{cases}L_{\nu} u=0, & x \geqslant 0, t \geqslant 0, \\ u(\mathbf{X}, 0)=0, & x \geqslant 0, \\ u(0, \mathbf{y}, t)=g, & t \geqslant 0 .\end{cases}
$$

We approximate it in the slab $0 \leqslant x \leqslant x_{1}$ by the problem

$$
\begin{cases}L_{\nu} u_{n}=0, & 0 \leqslant x \leqslant x_{1}, \quad t \geqslant 0, \\ u_{n}(\mathbf{X}, 0)=0, & 0 \leqslant x \leqslant x_{1}, \\ u_{n}(0, \mathbf{y}, t)=g, & t \geqslant 0, \\ B_{n} u_{n}\left(x_{1}, \mathbf{y}, t\right)=0, & t \geqslant 0 .\end{cases}
$$

The principal result of this section is

THEOREM 3. The boundary condition $B_{n}$ has an accuracy of order $2 n$ in $\nu$ : the error is bounded in $L^{2}\left(R_{+} \times R^{n-1}\right)$ for any $x$ in $\left.] 0, x_{1}\right]$ by

$$
\left\{\begin{array}{l}
\left\|u-u_{n}\right\|(x) \leqslant \nu^{2 n} d\left(x_{1}, \nu\right)\left\|L^{n} u\right\|(x) \quad \text { for } n \geqslant 1, \\
\left\|u-u_{0}\right\|(x) \leqslant \nu d\left(x_{1}, \nu\right)\left(\left\|B_{y} u\right\|+\nu\left\|\Delta_{y} u\right\|\right)(x),
\end{array}\right.
$$

where $B_{y}$ is the transverse transport operator defined in (2.23), the operator $L$ is

$$
L=B_{y}^{2}+\Delta_{y}
$$

and the function $d\left(x_{1}, \nu\right)$ is given by

$$
d\left(x_{1}, \nu\right)=\frac{2}{1-\exp \left(-x_{1} / 2 \nu\right)} .
$$

Remark 2. The bound depends on $\nu$ and $x_{1}$. In order to keep it small, $x_{1}$ must remain large compared to $\nu$. 
Proof of Theorem 3. The main tool will again be the Fourier transform with respect to $t$ and $\mathbf{y}$. We denote by $v_{n}$ the error in the slab $0 \leqslant x \leqslant x_{1}$,

$$
v_{n}=u-u_{n} .
$$

Using Section 1, we can write the Fourier transforms $\hat{u}$ and $\hat{v}_{n}$ of $u$ and $v_{n}$ explicitly as

$$
\begin{aligned}
& \hat{u}=\hat{g} e^{\lambda x}, \\
& \hat{v}_{n}=\hat{a} e^{\lambda x}+\hat{b} e^{\lambda^{\prime} x} .
\end{aligned}
$$

The coefficients $\hat{a}$ and $\hat{b}$ are given by the boundary conditions at $x=0$ and $x=x_{1}$, and $\hat{v}_{n}$ can be expressed as

$$
\hat{v}_{n}=R_{n}\left(e^{\left(\lambda-\lambda^{\prime}\right) x_{1}}-e^{\left(\lambda-\lambda^{\prime}\right)\left(x_{1}-x\right)}\right) \hat{u},
$$

where $R_{n}$ is related to the error coefficient $q_{n}$, defined in (2.19), through

$$
R_{n}=\frac{-q_{n}}{1-q_{n} e^{\left(\lambda-\lambda^{\prime}\right) x_{1}}}
$$

Since the real part of $\lambda-\lambda^{\prime}$ is negative, we get immediately a first bound on $\hat{v}_{n}$,

$$
\left|\hat{v}_{n}\right| \leqslant 2\left|R_{n} \| \hat{u}\right| \text {. }
$$

A bound on $q_{n}$ will give a bound on $R_{n}$, and thereby complete the proof.

LEMMA 2. The following bounds hold for $q_{n}$ :

(i) $\left|q_{n}\right| \leqslant 1$;

(ii) $\left|q_{n}\right| \leqslant \nu^{2 n}\left((\omega+\mathbf{a} \cdot \mathbf{k})^{2}+|\mathbf{k}|^{2}\right)^{n}$.

Proof. Since $q_{n}$ is equal to $\left(q_{1}\right)^{n}$, we only need to prove the lemma when $n$ is equal to 1 . The value of $q_{1}$ is

$$
q_{1}=\frac{1+2 i \nu(\omega+\mathbf{a} \cdot \mathbf{k})-\delta^{1 / 2}}{1+2 i \nu(\omega+\mathbf{a} \cdot \mathbf{k})+\delta^{1 / 2}} .
$$

We denote by $\alpha$ and $\beta$ the real and imaginary parts of $q_{1} ; \alpha$ is greater than one. It is easy to see that

$$
\left|q_{1}\right|=(\alpha-1) /(\alpha+1),
$$

which proves part (i). We note that

$$
\nu^{2}\left((\omega+\mathbf{a} \cdot \mathbf{k})^{2}+|\mathbf{k}|^{2}\right)=\left(\alpha^{2}-1\right)\left(\beta^{2}+1\right) / 4
$$

which leads to

$$
\frac{\left|q_{1}\right|}{\nu^{2}\left((\omega+\mathbf{a} \cdot \mathbf{k})^{2}+|\mathbf{k}|^{2}\right)}=\frac{4}{(\alpha+1)^{2}\left(\beta^{2}+1\right)} \leqslant 1,
$$

and thus finishes the proof of part (ii).

Using this lemma, we can bound $R_{n}$ by

$$
\left|R_{n}\right| \leqslant \frac{\nu^{2 n}\left((\omega+\mathbf{a} \cdot \mathbf{k})^{2}+|\mathbf{k}|^{2}\right)^{n}}{1-\exp \left(-x_{1} / 2 \nu\right)}
$$


We use this in (4.8), and obtain

$$
\left|\hat{v}_{n}\right| \leqslant d\left(x_{1}, \nu\right) \nu^{2 n}\left|F\left(L^{n} u\right)\right|
$$

where $F$ is the Fourier transform in $t$ and y. Parseval's theorem now completes the proof.

The estimate for condition $B_{0}$ is obtained in the same way.

Remark 3. The use of the Fourier transform in time is only formal here. But one can justify it by using the Laplace transform. The term $i \omega$ is replaced by $s$, with $\operatorname{Re} s \geqslant 0$. One easily proves that the denominator of $R_{n}$ cannot be zero. Then $\hat{u}_{n}$ is analytic in the halfspace $\operatorname{Re} s \geqslant 0$, and the Paley-Wiener theorem allows us to take the limit when $\operatorname{Re} s$ goes to zero.

5. One-Dimensional Numerical Experiments. We deal here with problem (4.1) in $R_{+} \times[0, T]$, and problem (4.2) in $[0,1] \times[0, T]$. We shall consider only the boundary operators $B_{0}, B_{1}$ and $B_{2}$. The initial value is chosen to be zero, and the boundary value at point $x=0$ is (cf. Figure 1)

$$
g(t)=\sin t / \sqrt{\left(t^{2}+1\right)} \text {. }
$$

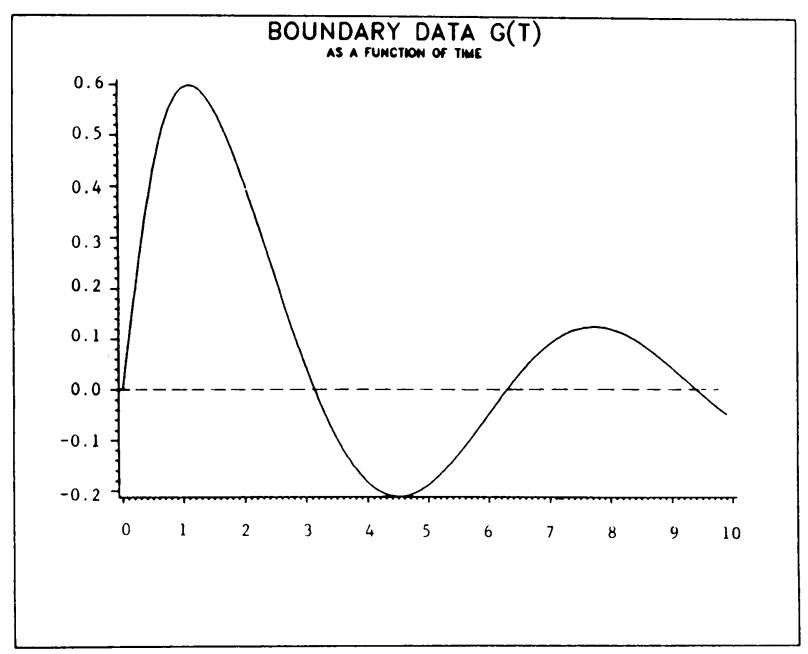

FigURE 1

Boundary data

We do not have the exact solution to problem (4.1) in closed form. But it can be observed that the reflection due to the boundary condition affects the solution only near the artificial boundary. Thus, we shall call here "exact" solution the computed solution in a larger domain, with a "good" boundary condition. For practical purposes, we take as the larger domain $[0,2] \times[0, T]$ and the second-order boundary condition $B_{2}$ at $x=2$.

We use second-order finite difference schemes in time and space. We recall some notations:

* $u_{j}^{n}$ approximates $u\left(x_{j}, t^{n}\right)$ on the grid $\left(x_{j}, t^{n}\right), 0 \leqslant j \leqslant J, 0 \leqslant n \leqslant N, x_{j}=j \Delta x$, $t^{n}=n \Delta t$. 
* The operators $D_{+}, D_{-}$, and $D_{0}$ denote forward, backward and centered differences, respectively. $S_{+}, S_{-}$and $S_{0}$ are forward, backward and centered sums; for example,

$$
D_{+}^{t} u_{j}^{n}=\left(u_{j}^{n+1}-u_{j}^{n}\right) / \Delta t, \quad S_{+}^{t} u_{j}^{n}=\left(u_{j}^{n+1}+u_{j}^{n}\right) / 2 .
$$

The operator $L_{\nu}$ is approximated by the following discrete scheme, derived from the Crank-Nicolson scheme,

$$
L^{d}=D_{+}^{t}+D_{0}^{x} S_{+}^{t}-\nu D_{+}^{x} D_{-}^{x} S_{+}^{t} .
$$

This scheme is implicit, has order two in time and space, and is unconditionally stable.

The operator $B_{0}$ is approximated by

$$
B_{0}^{d}=D_{-}^{x} .
$$

For the operator $B_{1}$, we use the transport part of operator $L^{d}$ and introduce a virtual point. This yields

$$
B_{1}^{d}=D_{+}^{t}+D_{-}^{x} S_{+}^{t} .
$$

Using the characterization of $B_{2}$ given by Lemma 1 , the second-order boundary condition is approximated by

$$
B_{2}^{d} u_{J}^{n}=\left(D_{+}^{t} D_{-}^{t}+2 D_{0}^{t} D_{-}^{x}\right) u_{J}^{n}+D_{+}^{x} D_{-}^{x} S_{0}^{t} u_{J-1}^{n}=0 .
$$

For any of these boundary conditions, the interior scheme together with the boundary scheme is stable and has order two in time and space.

The mesh sizes are taken equal to $\Delta t=\Delta x=0.001$.

Since we are dealing with parabolic equations, the maximum principle asserts that the largest error occurs at point $x$ equal to 1 . Hence Figures 2-4 show the "exact" solution and the error on the boundary for the various boundary conditions, as

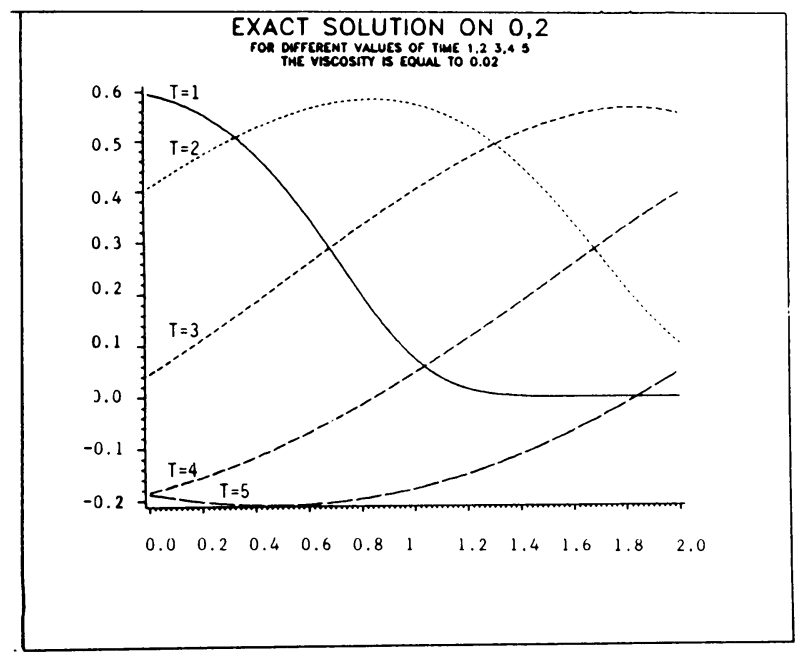

Figure 2

"exact" solution on $[0,2]$ for a viscosity equal to 0.02 ;

time varies from 1 to 5 


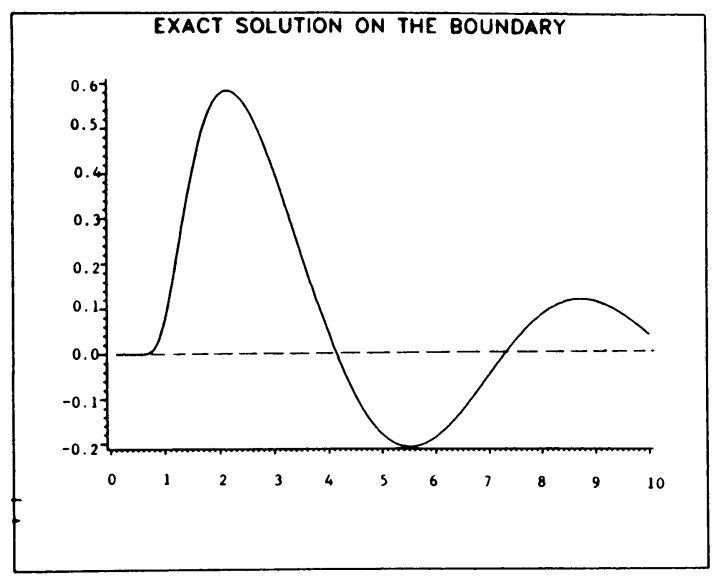

FIGURE 3

Exact solution on the boundary.

The viscosity is equal to 0.02 .
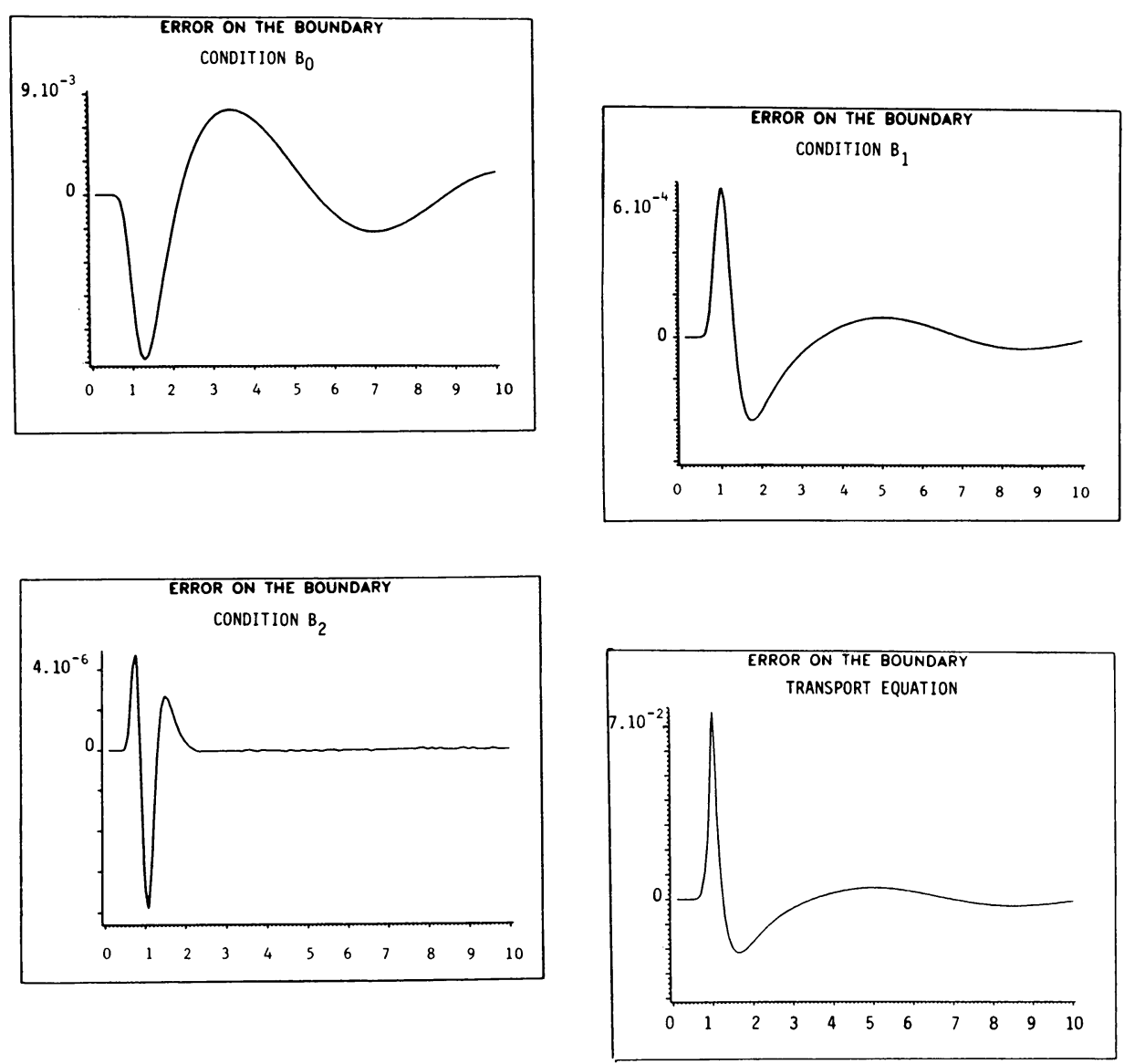

FIGURE 4

Errors on the boundary as functions of time.

The viscosity is equal to 0.02 
TABLE 1

$L^{2}$-norm in time of the error on the boundary

\begin{tabular}{|c|c|c|c|}
\hline viscosity & $B_{0}$ & $B_{1}$ & $B_{2}$ \\
\hline 0.002 & $0.210^{-2}$ & $0.810^{-5}$ & $0.710^{-8}$ \\
\hline 0.004 & $0.410^{-2}$ & $0.310^{-4}$ & $0.410^{-7}$ \\
\hline 0.006 & $0.510^{-2}$ & $0.610^{-4}$ & $0.110^{-6}$ \\
\hline 0.008 & $0.710^{-2}$ & $0.110^{-3}$ & $0.310^{-6}$ \\
\hline 0.01 & $0.810^{-2}$ & $0.210^{-3}$ & $0.610^{-6}$ \\
\hline 0.02 & $0.210^{-1}$ & $0.510^{-3}$ & $0.410^{-5}$ \\
\hline 0.04 & $0.310^{-1}$ & $0.210^{-2}$ & $0.310^{-4}$ \\
\hline 0.06 & $0.410^{-1}$ & $0.310^{-2}$ & $0.810^{-4}$ \\
\hline 0.08 & $0.510^{-1}$ & $0.510^{-2}$ & $0.210^{-3}$ \\
\hline 0.1 & $0.610^{-1}$ & $0.810^{-2}$ & $0.310^{-3}$ \\
\hline
\end{tabular}

functions of time. The last curve represents the error when replacing the diffusion equation by the transport equation in the domain, i.e., when ignoring the term $\nu \Delta u$.

One can see that the error oscillates and decreases in time, as does the exact solution. Moreover, the second-order condition $B_{2}$ produces the smallest error, while $B_{0}$ produces the largest. All these errors are smaller than the one produced by neglecting the diffusion in the equation.

Table 1 gives the $L^{2}$-norm of the error on the boundary, for different values of $\nu$ increasing from 0.002 to 0.1 .

To show the dependence in $\nu$, we plot in Figure 5 the logarithm of the $L^{2}$-error as a function of the logarithm of the viscosity.

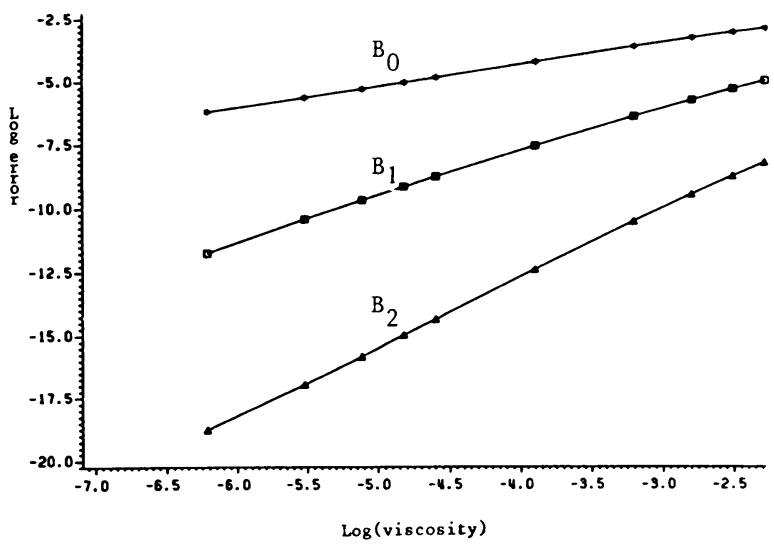

FIGURE 5

Logarithmic plot of $L^{2}$-error on boundary 
It can be seen that for $B_{0}$ and $B_{1}$ the dependence is linear in $\nu$, respectively quadratic, as stated in Theorem 2 , or even better. For the second-order condition, it is of fourth order in $\nu$ when $\nu$ is not too small. This of course is related to the size of $\Delta t$ and $\Delta x$ : the schemes have order two, and the error cannot become much smaller than $\Delta x^{2}$ and $\Delta t^{2}$.

6. Left-Hand Boundary Conditions. We are now formulating boundary conditions on the left wall $x_{2}$. In the same way as in Section 2, the transparent boundary condition is

$$
\frac{\partial \hat{u}}{\partial x}-\lambda^{\prime} \hat{u}=0
$$

where $\lambda^{\prime}$ is given by (2.10). In particular, we have

$$
\lambda^{\prime}=1 / \nu-\lambda .
$$

With this formula, we can approximate $\lambda^{\prime}$ by using the approximations to $\lambda$.

Thus, the first approximation to $\lambda^{\prime}$ is $\lambda_{0}^{\prime}=1 / \nu$, which gives the boundary condition

$$
B_{0}^{\prime} u=\nu \frac{\partial u}{\partial x}-u
$$

If $\lambda_{n}$ is the approximation to $\lambda$ given in Subsection 2.2, we define an approximation to $\lambda^{\prime}$ by

$$
\lambda_{n}^{\prime}=1 / \nu-\lambda_{n}, \quad n \geqslant 1 \text {. }
$$

Then $\lambda_{n}^{\prime}$ is an approximation of order $\nu^{2 n-1}$ to $\lambda^{\prime}$, and leads to an inhomogeneous boundary condition. The boundary operator $B_{n}^{\prime}$ can be written as in Lemma 1,

$$
B_{n}^{\prime}=\left(B_{1}^{\prime}\right)^{n}
$$

where the first-order operator $B_{1}^{\prime}$ is defined by

$$
B_{1}^{\prime} u=\nu\left(u_{x}-u_{t}-\mathbf{a} \cdot \nabla u\right)-u .
$$

From the recursion formula (6.5) one can deduce, as in the previous sections, that:

* The associated initial boundary value problems in the halfspace $x \geqslant 0$ are well-posed.

* The boundary condition $B_{n}^{\prime}$ has accuracy of order $2 n$ in $\nu$.

Conclusion. We have developed two families of artificial boundary conditions for the linear advection diffusion equation, when the viscosity is small. They lead to well-posed initial boundary value problems and produce errors which are powers of $\nu$. The numerical experiments confirm the theoretical study.

Acknowledgments. I thank M. Schatzman for suggesting this work and for providing helpful comments. 
1. B. ENGQUIST \& A. MAJDA, "Radiation boundary conditions for acoustic and elastic wave calculations," Comm. Pure Appl. Math., v. 32, 1979, pp. 313-357.

2. B. ENGQUIST \& A. MAJDA, "Numerical radiation boundary conditions for unsteady transsonic flow," J. Comput. Phys., v. 40, 1981, pp. 91-103.

3. G. W. Hedstrom, "Nonreflecting boundary conditions for nonlinear hyperbolic systems," $J$. Comput. Phys., v. 30, 1979, pp. 222-237.

4. A. N. Khovanskil, The Application of Continued Fractions and Their Generalizations to Problems in Approximation Theory, Noordhoff, Groningen, 1963.

5. J. L. Lions \& E. Magenes, Problèmes aux Limites Non Homogènes et Applications, Dunod, Paris, 1968.

6. G. J. MCRAE et al., "Numerical solution of the atmospheric diffusion equation for chemically reacting flows," J. Comput. Phys., v. 45, 1982, pp. 1-42.

7. R. Temam, Navier-Stokes Equations. Theory and Numerical Analysis, North-Holland, Amsterdam, 1977. 